\title{
Evolución de la prevalencia de enteroparasitosis en la ciudad de Talca, Región del Maule, Chile
}

\author{
Sylvia Vidal F., Lorena Toloza M. y Beatriz Cancino F.
}

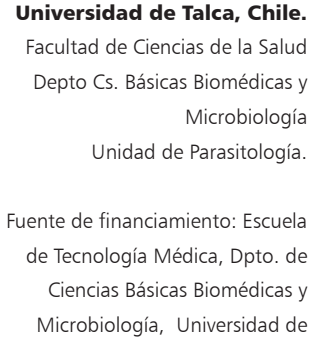

Fuente de financiamiento: Escuela de Tecnología Médica, Dpto. de

Ciencias Básicas Biomédicas y Microbiología, Universidad de

Los autores declaran que no existe conflicto de intereses en el presente trabajo.

Recibido: 26 de noviembre de 2009 Aceptado: 28 de abril de 2010

Correspondencia a: Sylvia Vidal F. svidal@utalca.cl

\section{Introducción}

Las enteroparasitosis intestinales representan un problema de salud pública, tanto en zonas rurales como urbanas de países en vías de desarrollo. Éstas se originan debido a la ausencia de medidas sanitarias básicas como alcantarillado y eliminación de aguas contaminadas, problemas de hacinamiento en la población y malos hábitos higiénicos ${ }^{1,2}$.

La alta incidencia de infección por parásitos intestinales y poliparasitismo afecta la salud de los individuos, pudiendo causar deficiencia en el aprendizaje y función cognitiva. Principalmente los niños son los más afectados por la no incorporación de hábitos higiénicos o la mal nutrición, en especial en países con menor desarrollo o con altos índices de ruralidad de la población ${ }^{3-6}$.

En el curso de los últimos años, las parasitosis han cambiado su curso clásico con el mejoramiento de las medidas sanitarias: los parásitos macroscópicos se han ido erradicando como causa de enfermedad intestinal y los protozoos han ido aumentando en número. Además, han aparecido otros elementos parasitarios denominados parásitos emergentes los que han producido un cambio conceptual de aplicación práctica en la epidemiología, patogenia y tratamiento de las enfermedades ${ }^{7-8}$.

Hoy en día se acepta que parásitos considerados exclusivos de una zona geográfica, por razones de migración y cambios climáticos se puedan encontrar en otras zonas.
Chile por su parte, presenta en concordancia con sus características geográficas y de desarrollo urbano, una diversidad de situaciones con respecto a cada enteroparásito; algunos de ellos, especialmente las geo-helmintiasis, han tendido prácticamente a desaparecer de las áreas urbanas del centro del país. Sin embargo, mantienen cierta presencia en el territorio sur, donde las condiciones del terreno aún le son favorables. Por otro lado, algunos parásitos cuya dependencia de las características ambientales es menor o mínima, tienen una prevalencia significativa en todo el país, sin mayores variaciones en el tiempo, pese al mejoramiento en los niveles de vida.

Entre los elementos protozoarios emergentes comúnmente pesquisados, Blastocystis hominis presenta una alta prevalencia en los países en vías de desarrollo, llegando incluso a cifras cercanas a $50 \% 0^{9-14}$. A pesar de ser uno de los protozoarios más reportados en muestras fecales humanas, la epidemiología de $B$. hominis aún está en estudio. Anteriormente considerado un parásito saprófito, hoy se acepta que en muchas circunstancias puede tener un rol patógeno y debe ser tratado si persiste la sintomatología, habiéndose descartado la presencia de otros patógenos ${ }^{15-20}$.

Por otra parte, parásitos como Cyclospora cayetanensis, Isospora belli, Giardia lamblia, Endolimax nana y Chilomastix mesnili, han aumentado, principalmente en los pacientes inmunocomprometidos ${ }^{21}$.

En Chile, estudios epidemiológicos efectuados en la última década indican que las infecciones parasitarias 
intestinales continúan siendo endémicas, afectando, con diversa prevalencia, a la población urbana y rural del país ${ }^{22-23}$.

La Región del Maule cuenta con índices de ruralidad que van desde $4,23 \%$ en la provincia de Talca hasta $71,13 \%$ en la comuna de San Clemente, ubicándose como una de las regiones con más altos índices de ruralidad nacional ${ }^{24}$.

El objetivo de este trabajo fue evaluar la evolución, a través del tiempo, de la prevalencia de las enteroparasitosis, en un segmento importante de la población como son los preescolares y escolares básicos provenientes de la zona periférica de Talca y zonas rurales de la Región del Maule, Chile.

\section{Material y Método}

Se realizó un estudio descriptivo retrospectivo a partir de los resultados obtenidos en los exámenes coproparasitológicos seriados procesados mediante el método de Burrows ${ }^{25}$, y técnica de Gram $^{26}$, en el Laboratorio de Parasitología de la Universidad de Talca, entre los años 1980 y 2008.

La población estudiada correspondió a niños, aparentemente sanos, de colegios básicos municipalizados y jardines infantiles pertenecientes a la Junta Nacional de Jardines infantiles (JUNJI), provenientes de la zona periférica de la ciudad de Talca y zonas rurales de la Región del Maule. Algunas de las provincias estudiadas fueron Romeral, Molina, Cumpeo, Sagrada Familia, San Rafael, San Clemente, Pelarco, Maule, Chanco, Pencahue, Villa Alegre, Yerbas Buenas, entre otras. De esta forma, se llegó a analizar un total de 68.142 muestras. Todas las muestras fueron recolectadas en el período primavera-verano.
Recolección de muestras. Se recolectaron tres muestras de deposición, de días no consecutivos en un frasco "boca ancha”, con solución de fenol, alcohol, formalina (PAF) Las muestras fueron almacenadas a temperatura ambiente, en un lugar fresco, seco y alejado del sol, por un máximo de 7 días hasta ser procesadas por el método de Burrows ${ }^{25}$. Además de tomó a cada niño test de Graham ${ }^{26}$, el cual fue observado en un microscopio con aumento de 10 y $40 \mathrm{X}$.

\section{Resultados}

En la Tabla 1 y Figura 1 se muestra la prevalencia de enteroparasitosis durante el período 1980-2008, los

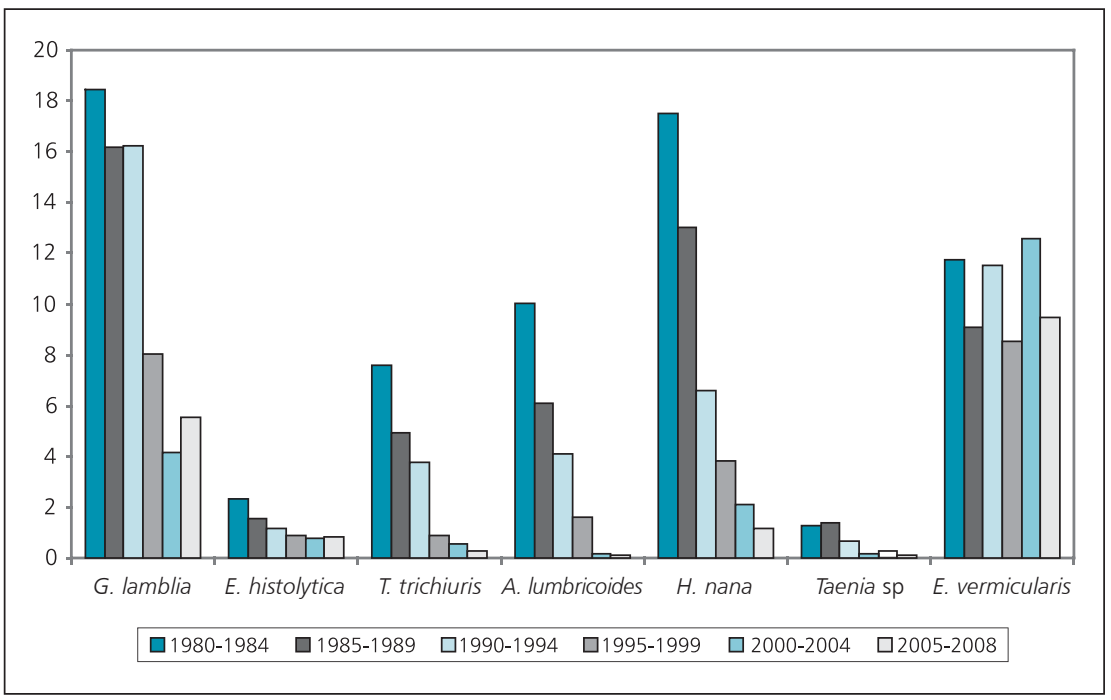

Figura 1. Prevalencia quinquenal de enteroparasitosis causadas por patógenos en escolares y preescolares de la ciudad de Talca, en el período 1980-2008. Expresada en porcentaje.

\begin{tabular}{|c|c|c|c|c|c|c|c|c|c|c|c|c|c|c|c|}
\hline \multirow{2}{*}{$\begin{array}{l}\text { Período } \\
\text { Años }\end{array}$} & \multirow{2}{*}{$\begin{array}{c}\text { Niños } \\
\text { estudiados }\end{array}$} & \multicolumn{2}{|c|}{ G. lamblia } & \multicolumn{2}{|c|}{ E. histolytica } & \multicolumn{2}{|c|}{ T. trichiuris } & \multicolumn{2}{|c|}{ A. lumbricoides } & \multicolumn{2}{|c|}{ H. nana } & \multicolumn{2}{|c|}{ Taenia sp } & \multicolumn{2}{|c|}{ E. vermicularis } \\
\hline & & $\mathbf{n}$ & $\%$ & $\mathbf{n}$ & $\%$ & $\mathbf{n}$ & $\%$ & $\mathbf{n}$ & $\%$ & $\mathbf{n}$ & $\%$ & $\mathbf{n}$ & $\%$ & $\mathbf{n}$ & $\%$ \\
\hline $1980-84$ & 10.205 & 1.883 & 18,45 & 235 & 2,3 & 776 & 7,6 & 1.024 & 10,03 & 1.789 & 17,53 & 126 & 1,25 & 1.200 & 11,75 \\
\hline $1985-89$ & 12.010 & 1.945 & 16,19 & 185 & 1,54 & 595 & 4,95 & 732 & 6,09 & 1.564 & 13,02 & 165 & 1,37 & 1.098 & 9,11 \\
\hline 1990-94 & 11.680 & 1.898 & 16,25 & 135 & 1,15 & 439 & 3,75 & 479 & 4,1 & 773 & 6,61 & 78 & 0,66 & 1.350 & 11,55 \\
\hline $1995-99$ & 11.810 & 952 & 8,06 & 103 & 0,87 & 102 & 0,86 & 189 & 1,6 & 451 & 3,81 & 21 & 0,17 & 1.008 & 8,53 \\
\hline $2000-04$ & 12.050 & 523 & 4,18 & 97 & 0,8 & 64 & 0,53 & 24 & 0,19 & 257 & 2,13 & 35 & 0,29 & 1.537 & 12,75 \\
\hline 2005-08 & 10.387 & 575 & 5,53 & 85 & 0,81 & 32 & 0,3 & 12 & 0,11 & 124 & 1,19 & 13 & 0,12 & 987 & 9,5 \\
\hline Totales & 68.142 & 7.776 & 11,44 & 840 & 1,25 & 2.008 & 2,95 & 2.460 & 3,69 & 4.958 & 7,38 & 438 & 0,64 & 7.180 & 10,53 \\
\hline \multicolumn{2}{|l|}{ D.E* } & \multicolumn{2}{|c|}{687,6} & \multicolumn{2}{|c|}{58,9} & \multicolumn{2}{|c|}{313,8} & \multicolumn{2}{|c|}{410,3} & \multicolumn{2}{|c|}{697,4} & \multicolumn{2}{|c|}{61,7} & \multicolumn{2}{|c|}{213,8} \\
\hline
\end{tabular}


Tabla 2. Prevalencia quinquenal de infecciones Enteroparasitarias, producidas por comensales en preescolares y escolares de Talca,

\begin{tabular}{|c|c|c|c|c|c|c|c|c|c|c|c|}
\hline \multirow{2}{*}{$\begin{array}{l}\text { Período } \\
\text { Años }\end{array}$} & \multirow{2}{*}{$\begin{array}{c}\text { Niños } \\
\text { estudiados }\end{array}$} & \multicolumn{2}{|c|}{ E. coli } & \multicolumn{2}{|c|}{ B. hominis } & \multicolumn{2}{|c|}{ I. butschlii } & \multicolumn{2}{|c|}{ E. nana } & \multicolumn{2}{|c|}{ Ch. mesnili } \\
\hline & & $\mathbf{n}$ & $\%$ & $\mathbf{n}$ & $\%$ & $\mathbf{n}$ & $\%$ & $\mathbf{n}$ & $\%$ & $\mathbf{n}$ & $\%$ \\
\hline 1980-84 & 10.205 & 2.083 & 20,41 & $N . R^{* *}$ & & 1.432 & 14,03 & 2.680 & 26,26 & 1.989 & 19,49 \\
\hline 1985-89 & 12.010 & 2.070 & 17,24 & $N \cdot R^{* *}$ & & 1.570 & 13,07 & 2.364 & 19,68 & 1.745 & 14,52 \\
\hline 1990-94 & 11.680 & 1.390 & 11,9 & 890 & 7,61 & 1.346 & 11,52 & 2.097 & 17,95 & 1.650 & 14,12 \\
\hline 1995-99 & 11.810 & 1.075 & 9,1 & 1.025 & 8,68 & 1.250 & 10,58 & 1.973 & 16,7 & 1.450 & 12,27 \\
\hline $2000-04$ & 12.050 & 2.030 & 16,85 & 6.312 & 52,38 & 1.005 & 8,34 & 1.042 & 8,64 & 9,75 & 8,09 \\
\hline 2005-07 & 10.387 & 1.054 & 10,15 & 7.580 & 72,97 & 1.129 & 10,87 & 1.054 & 10,14 & 645 & 6,2 \\
\hline Totales & 68.142 & 9.702 & 14,27 & 15.807 & 35,41 & 7.732 & 11,4 & 11.210 & 16,56 & 7.489 & 12,45 \\
\hline D.E* & & \multicolumn{2}{|c|}{501} & \multicolumn{2}{|c|}{$3.496,4$} & \multicolumn{2}{|c|}{205,2} & \multicolumn{2}{|c|}{680,2} & \multicolumn{2}{|c|}{761} \\
\hline
\end{tabular}

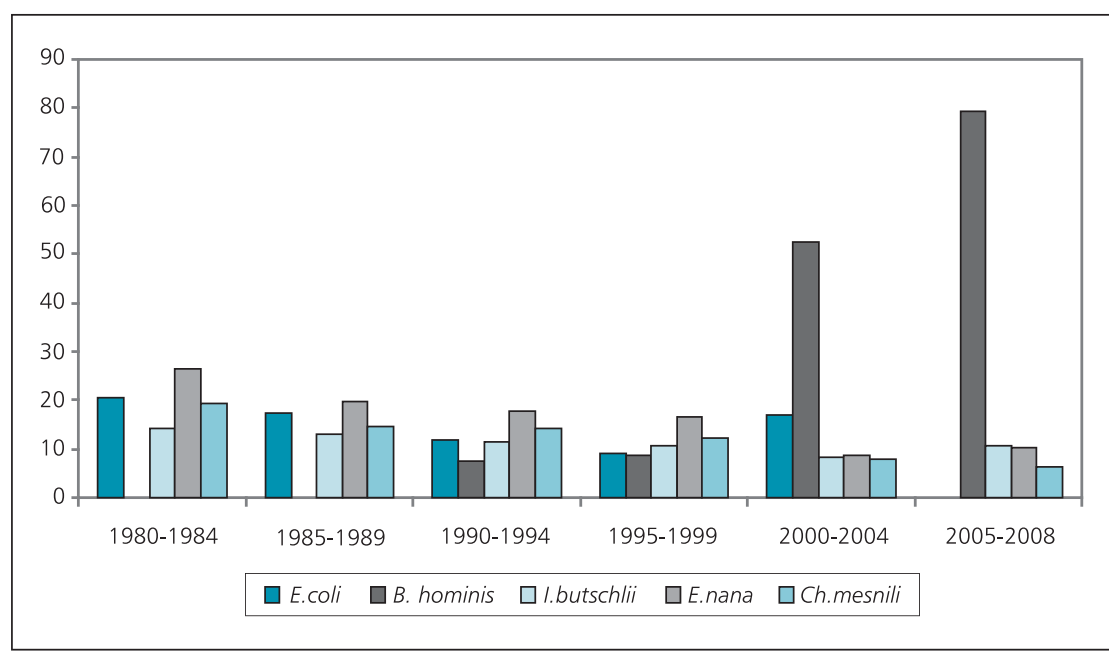

Figura 2. Prevalencia quinquenal de enteroparasitosis causadas por comensales en escolares y preescolares de la ciudad de Talca, en el período 1980-2008. Expresada en porcentaje.

años fueron agrupados en quinquenios con un número de 68.142 muestras analizadas, correspondientes a niños de localidades rurales de la Región del Maule; se observa un total de $37,8 \%$ de niños infectados por parásitos patógenos. Al analizarlos en detalle se observa un franco descenso a través del tiempo en G. lamblia, Entamoeba histolytica, Trichocephalos trichiuris, Ascaris lumbricoides, Hymenolepis nana y Taenia sp.

La Tabla 2 y Figura 2 muestran el comportamiento de los parásitos comensales a través del tiempo, en los cuales vemos una disminución no tan acentuada, como ocurre en los parásitos patógenos,

En la Tabla 3 y Figura 3 observamos la prevalencia total de la población, calculada en $76,2 \%$ de niños infec- tados con parásitos, ya fuesen comensales o patógenos. En relación al número de elementos encontrados en las muestras se observa que, a medida que transcurre el tiempo, el poliparasitismo disminuyó notablemente desde 64,5 a 9,6\%; en forma inversa, el monoparasitismo aumentó desde 10,0 a $35,5 \%$.

\section{Discusión}

Al analizar en detalle los resultados por evento de infección de cada elemento parasitario, observamos un franco descenso de patógenos a través del tiempo con porcentajes de que varían desde $9,8 \%$ de portación promedio parasitaria en el primer período (1980-1984) a 2,5\% en el último período (2005-2008). Específicamente se observó un marcado descenso, en la portación de G. lamblia, T. trichiura, A. lumbricoides, H. nana, E. histolytica y Taenia sp. La mantención en las cifras de enterobiasis se podría explica porque Enterobius vermicularis es un parásito cosmopolita que, por su particular biología, contribuye a la creación de focos de contaminación cercana al hospedero parasitado por su fácil diseminación y contagio. Además es frecuente en distintas zonas templadas, en forma independiente del nivel sanitario del individuo. Se condice este hallazgo con otras publicaciones, tanto nacionales como extranjeras $^{27-31}$. En relación a los parásitos comensales éstos presentan también una alta ocurrencia y una variabilidad no tan marcada como los patógenos, en los casos de E. coli, Iodamoeba bütschlii, E. nana y $C$. mesnili, con una porcentaje promedio de portación de $18,3 \%$ para el primer quinquenio (1985-1989) a $22,1 \%$ para el último período (2005-2008). Influye en esta cifra un significativo aumento en la portación de $B$. hominis, 
Tabla 3. Prevalencia quinquenal del № de parásitos encontrados en los niños analizados durante el período 1980-2008

\begin{tabular}{|c|c|c|c|c|c|c|c|}
\hline \multirow{2}{*}{$\begin{array}{l}\text { Período } \\
\text { Años }\end{array}$} & \multirow{2}{*}{$\begin{array}{c}\text { Niños } \\
\text { estudiados }\end{array}$} & \multicolumn{2}{|c|}{ Un parásito } & \multicolumn{2}{|c|}{ Dos parásitos } & \multicolumn{2}{|c|}{ Tres ó mas parásitos } \\
\hline & & $\mathbf{n}$ & $\%$ & $\mathbf{n}$ & $\%$ & $\mathbf{n}$ & $\%$ \\
\hline $1980-84$ & 10.205 & 1.025 & 10,04 & 2.056 & 20,15 & 6.589 & 64,57 \\
\hline $1985-89$ & 12.010 & 1.760 & 14,65 & 2.870 & 23,9 & 5.340 & 44,46 \\
\hline 1990-94 & 11.680 & 2.789 & 23,88 & 2.360 & 20,21 & 4.321 & 36,99 \\
\hline 1995-99 & 11.810 & 3.799 & 32,17 & 2.256 & 19,1 & 2.870 & 24,3 \\
\hline $2000-04$ & 12.050 & 3.350 & 27,8 & 2.267 & 18,81 & 1.438 & 11,93 \\
\hline 2005-07 & 10.387 & 3.690 & 35,53 & 2.005 & 19,3 & 985 & 9,48 \\
\hline Totales & 68.142 & 16.413 & 24,01 & 13.814 & 20,24 & 21.543 & 31,96 \\
\hline D. E* & & \multicolumn{2}{|c|}{$1.122,5$} & \multicolumn{2}{|c|}{152} & \multicolumn{2}{|c|}{2214,8} \\
\hline
\end{tabular}

presentando éste el valor más significativo del estudio (35,4\% globalmente). Cabe destacar que sólo comenzó a detectarse en la década del 90, no existen registros de datos anteriores a esta fecha. Las cifras encontradas en la literatura científica ${ }^{32-34}$ en las décadas del 80 y 90 en cada quinquenio, son cercanas a $10 \%$; no obstante, en los últimos años se ha visto un aumento explosivo de blastocistosis, encontrándose en el presente estudio cifras de 72,9\% entre los años 2005 y 2008 . Este valor concuerda con un artículo publicado en Chile, que indica que la blastocistosis es la parasitosis más frecuente, con un porcentaje que varía entre 41,3 y $62,3 \%$ de los estudiados $^{34}$. Además se correlaciona con hallazgos en el extranjero ${ }^{4,8,22,23}$. Esto podría deberse, por una parte, a la no descripción de estos elementos en los años 1980 y al mayor conocimiento y adiestramiento de los laboratorios para reconocer este protozoo hoy.

Podríamos concluir que el descenso observado en la mayoría de las enteroparasitosis en los últimos quinquenios, tiene su origen en el mejor saneamiento básico y cambio de los factores socioeconómicos y culturales, por ejemplo, la masiva potabilización de agua en lugares de la región, medidas específicas de cloración y campañas educativas como la efectuada contra el cólera ${ }^{33}$ a partir del año 19917,35,36. En el caso específico de las teniasis también incide el exigente control veterinario en las plantas faenadoras, dadas las exigencias del mercado, todo esto unido a una mayor educación de la población en general.

Agradecimientos. A todos y cada uno de los alumnos de la carrera de Tecnología Médica de la Universidad de Talca, que realizaron el curso de Parasitología II en el período 1980 a 2008.

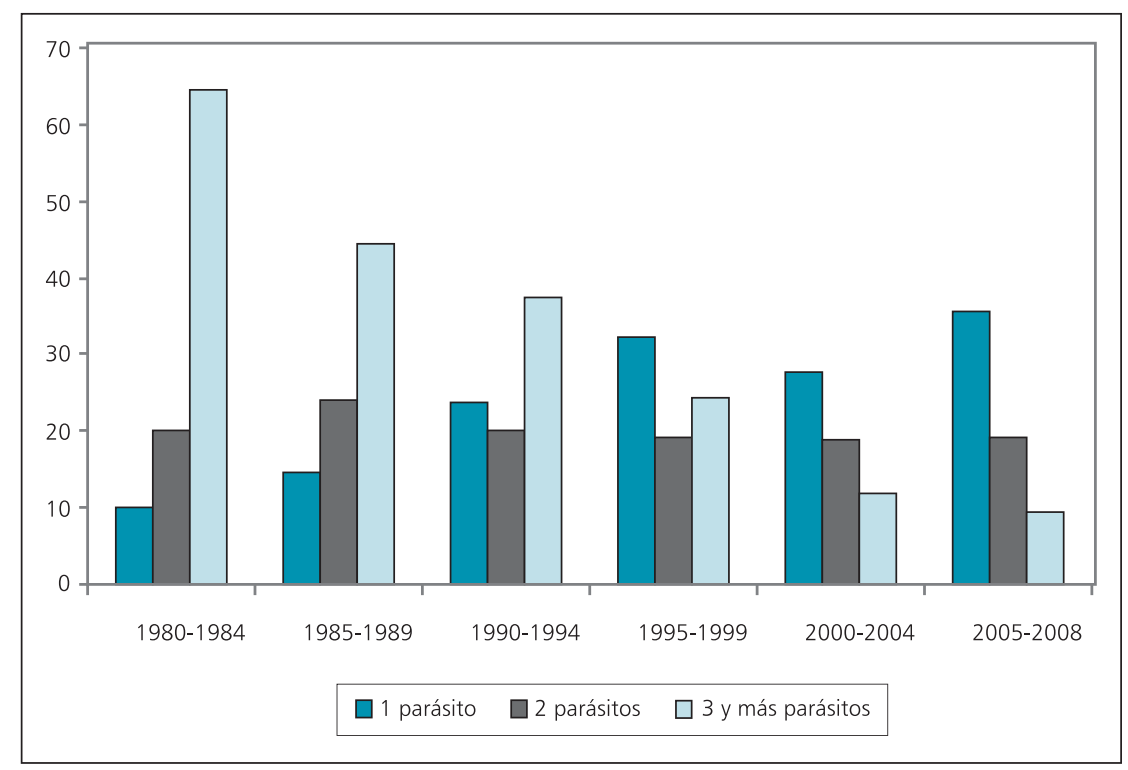

Figura 3. Diferencia entre monoparasitismo y poliparasitismo en el período 1980 a 2008. Expresada en porcentaje.

\section{Resumen}

Se realizó un estudio descriptivo y retrospectivo de prevalencia de enteroparasitosis en preescolares y escolares de la zona periférica de la ciudad de Talca y zonas rurales de la región del Maule, a partir de los resultados obtenidos en los exámenes copro-parasitológicos seriados y test de Graham, procesados en el Laboratorio de Parasitología de la Universidad de Talca, durante el período comprendido entre los años 1980 y 2008. Observamos la prevalencia total de la población calculada en $76,2 \%$ de niños infec- 
tados con parásitos, ya fuesen comensales o patógenos. Se observó un franco descenso, de $9,8 \%$ a $2,5 \%$, de enteroparasitosis causadas por los siguientes patógenos: Giardia lamblia, Entamoeba histolytica, Trichocephalos trichiuris, Ascaris lumbricoides, Hymenolepis nana, Taenia sp. Las enteroparasitosis por comensales como Entamoeba coli, Iodamoeba bütschlii, Endolimax nana y Chilomastix mesnili experimentaron un leve descenso. A pesar de esta disminución, los parásitos comensales muestran un aumento a través del tiempo, dado por el significativo aumento en la portación de Blastocystis hominis (de 7,6 a 72,9\%). Se observó además un cambio en la portación de poliparasitosis (de 64,5 a 9,6\%) y monoparasitosis (de 10,0 a 35,5\%).

\section{Referencias}

1.- Rivera de R Z, Acevedo Z, Casanova C, Hernández S, Malaspina A. Enteroparasitosis en escolares de dos unidades educativas rurales del municipio La Cañada: Estado Zulia-Venezuela. 1996; 24 (3): 151-77.

2.- Milano A, Oscherov E, Palladito A, Bar A. Enteroparasitosis infantil en un área urbana del nordeste Argentino. Medicina (Buenos Aires) 2007; 67: 238-42

3.- Salomon M, Tonelli R, Borremans C, Bertello D, De Jong L, Jofré C, Enríquez V, et al. Prevalence of intestinal parasites in children living in Mendoza city, Argentina. Parasitol Latinoam 2007; 62 (1-2): 49-53.

4.- Mercado R, Castillo D, Muñoz V, Sandoval L, Jercic M, Gil L, et al. Infecciones por protozoos $\mathrm{y}$ helmintos intestinales en pre-escolares y escolares de la comuna de Colina, Santiago, Chile 2003. Parasitol Latinoam 2003; 58: 173-6.

5.- $\quad$ Marcos R L A, Maco F V, Iwashita T A. Prevalencia de parasitosis intestinal en niños del Valle del Mantaro, Jauja, Perú. Rev Med Hered 2002; 13 (3): 85-90.

6.- Zonta M, Navone G, Oyehenart E. Parasitosis intestinales en niños de edad preescolar y escolar: situación actual en poblaciones urbanas, periurbanas y rurales en Brandsen, Buenos Aires, Argentina. Parasitol Latinoam 2007; 62 (1-2): 54-60.

7.- Chen S H, Codoceo A, Carrasco O, Torres M. Enteroparasitosis en la población de la tercera edad consultante en centros médicos de la Pontificia Universidad Católica de Chile, 1997. Parasitol Día 1998; 22 (3-4): 114-6.

8.- Mercado P R, Schenone F H. Blastocistosis: Enteroparasitosis más frecuente en Chile. Rev Méd Chile 2004; 132 (8): 1015-6.

9.- Tassara O R. Enteroparasitosis: Realidad actual y manejo. Rev Chil Pediatr 1999; 70 (5): 441-5.

10.- Navarrete N, Torres P. Prevalencia de infección por protozoos y helmintos intestinales en escolares de un sector costero de la provincia de Valdivia. Bol Chil Parasitol 1994; 49: 79-80.

11.- Biolley M A, Hernández V, Rojas F, Rodríguez A. Protozoos intestinales en lactantes y preescolares de residencia urbana. Temuco IX Región. Parasitol Día 1995; 19: 252.

12.- Otto J, Musleh M, Pérez M. Enteroparasitosis en 40 grupos familiares de la localidad de Chauquear, Isla Puluqui, X Región de Chile, 1997. Parasitol Día 1998; 22: 11-2.

13.- Neira O P, Muñoz N, Carabelli M, Subrecaseaux B, Tardío M, Villalón T, et al. Enteroparasitosis en la V Región, Chile: estudio en escolares rurales de Santo Domingo, 1987. Bol Chil Parasitol 1990; 45: 24-7.

14.- Traviezo-Valles L, Triolo M M, Agobian G. Predominio de Blastocystis hominis sobre otros enteroparásitos en pacientes del municipio Palavecino, Estado Lara, Venezuela. Rev Cubana Med Trop 2006; 58 (1): 14-8.

15.- Denegri M. Blastocistocis, En: Atías A. Parasitología Médica, Tercera Edición, Santiago Chile, Editorial Mediterráneo 1998. pp 161-3.

16.- Babcock D, Houston R, Kumaki D, Shlim D. Blastocystis hominis in Kathmandu, Nepal. N Engl J Med 1985; 313 (22): 1419.

17.- Doyle P, Helgason M, Mathias G, Proctor E. Epidemiology and pathogenicity of Blastocystis hominis. J Clin Microbiol 1990; 28: 116-21.

18.- Al-Tawil S, Gilbert M, Gopalakrishna G, Langston C, Bommer K. Invasive Blastocystis hominis infection in a child. Arch Pediatr Adolesc Med 1994; 148: 882-5.

19.- Garavelli P, Libanore M. Blastocystis in immunodeficiency diseases. Rev Infect Dis 1990; 12: 158

20.- Chen, Shu-Hsing, García A, Torres M. Enteroparasitosis en el Hospital San Juan de Dios, Illapel, Chile. 1995. Parasitol Día 1998; 22 (3-4): 118-22.

21.- Abuin J, Marino D. Diarreas de origen parasitario en pacientes VIH (+). Disponible en: http://www.sasnac.org.ar/docs/ciencias clinicas/C/diarreas_de_origen_parasitario_en_ pacientes_hiv.pdf, (accedido 10 agosto 2009).

22.- Bórquez C, Lobato I, Montalvo M, Marchant P, Martínez P. Enteroparasitosis en niños escolares del Valle de Lluta. Arica-Chile. Parasitol Latinoamer 2004; 59 (3-4): 175-8.

23.- Torres P, Miranda J C, Flores L, Riquelme J, Franjola R, Pérez J, et al. Blastocistosis y otras infecciones por protozoos en comunidades humanas y ribereñas de la cuenca del río Valdivia, Chile.1992, Rev Inst Med Trop Sao Paulo 1992; 34 (6): 557-64

24.- Ficha comunal del sistema nacional de indicadores municipales. Disponible en: http: www.talca.cl. (accedido $1^{\circ}$ de agosto 2009).
25.- Muñoz V, Dorn L, Reyes H. Examen coproparasitológico: Evaluación de algunas modificaciones al método de Burrows. Parasitol Día 1984; 8: 107-11.

26.- Graham C F. A device for the diagnosis of Enterobius infection. Amer J Trop Med 1941; 21: 159-61.

27.- Markelll E, Voge M, John D. Los Nemátodes Intestinales, Markelll E, Voge M, John D. Parasitología Médica, sexta edición 1996, Madrid, Editorial Interamericana. Mc Graw Hill, pp: 219-47.

28.- Cazorla D J, Acosta M E, Zárraga A, Morales P. Estudio clínico-epidemiológico de enterobiasis en preescolares y escolares de Taratara, Estado Falcón, Venezuela. Parasitol Latinoam 2006; 61 (1-2): 43-53.

29.- Acosta M E, Cazorla D J, Garvett M. Enterobiasis en escolares de una población rural del Estado Falcón, Venezuela y su relación con el nivel socio-económico. Invest Clin 2002; 43 : 173-81.

30.- Devera R, Pérez C, Ramos Y. Enterobiasis en escolares de Ciudad Bolívar, Estado Bolívar, Venezuela. Bol Chil Parasitol 1998; 53: 14-8.

31.- Norhayati M, Hayati M, Oothuman P, Azzio O, Fatmah M S, Ismail G, et al. Enterobius vermicularis infection among children aged 1-8 years in a rural area of Malaysia. Southeast Asian J Trop Med Public Health 1994; 25: 494-7.

32.- Markell E. Editorial: Is there any reason to continue treating Blastocystis hominis infections? Clin Infect Dis 1995; 21(1): 104-5. Review.

33.- Laval E. El cólera en Chile (1886-1888). Rev Chil Infect 2003; 20 (Edición aniversario): 86-8.

34.- Barahona L, Maguiña C, Náquira C. Blastocistosis humana: Estudio prospectivo, sintomatología y factores epidemiológicos asociados. Rev Gastroenterol Perú 2003; 23 (1): 29-35.

35.- Szot J. Reseña de la salud pública maternoinfantil chilena durante los últimos 40 años: 1960-2000. Rev Chil Obstet Ginecol 2002; 67 (2): 129-35.

36.- Campaña de Salud, Verano saludable, Ministerio de Salud, Departamento de Epidemiología. Disponible en: http://epi.minsal.cl/epi/html/ frames/frame5.htm (accedido 10 de agosto 2009). 